\title{
Atomic-Scale Surface Demixing in a Eutectic Liquid BiSn Alloy
}

\section{Citation}

Shpyrko, Oleg G., Alexei Yu. Grigoriev, Reinhard Streitel, Diego Pontoni, Peter S. Pershan, Moshe Deutsch, Ben Ocko, Mati Meron, and Binhau Lin. 2005. Atomic-scale surface demixing in a eutectic liquid BiSn alloy. Physical Review Letters 95(106103).

\section{Published Version}

doi:10.1103/PhysRevLett.95.106103

\section{Permanent link}

http://nrs.harvard.edu/urn-3:HUL.InstRepos:4243717

\section{Terms of Use}

This article was downloaded from Harvard University's DASH repository, and is made available under the terms and conditions applicable to Other Posted Material, as set forth at http:// nrs.harvard.edu/urn-3:HUL.InstRepos:dash.current.terms-of-use\#LAA

\section{Share Your Story}

The Harvard community has made this article openly available.

Please share how this access benefits you. Submit a story.

Accessibility 


\title{
Atomic-Scale Surface Demixing in a Eutectic Liquid BiSn Alloy
}

\author{
Oleg G. Shpyrko, ${ }^{1, *}$ Alexei Yu. Grigoriev, ${ }^{1}$ Reinhard Streitel, ${ }^{1}$ Diego Pontoni, ${ }^{1}$ Peter S. Pershan, ${ }^{1}$ Moshe Deutsch, ${ }^{2}$ \\ Ben Ocko, ${ }^{3}$ Mati Meron, ${ }^{4}$ and Binhua Lin $^{4}$ \\ ${ }^{1}$ Department of Physics and DEAS, Harvard University, Cambridge, Massachusetts 02138, USA \\ ${ }^{2}$ Department of Physics, Bar-Ilan University, Ramat-Gan 52900, Israel \\ ${ }^{3}$ Department of Physics, Brookhaven National Laboratory, Upton, New York 11973, USA \\ ${ }^{4}$ CARS, University of Chicago, Chicago, Illinois 60637, USA
}

(Received 17 March 2005; published 1 September 2005)

\begin{abstract}
Resonant $\mathrm{x}$-ray reflectivity of the surface of the liquid phase of the $\mathrm{Bi}_{43} \mathrm{Sn}_{57}$ eutectic alloy reveals atomic-scale demixing extending over three near-surface atomic layers. Because of the absence of an underlying atomic lattice which typically defines adsorption in crystalline alloys, studies of adsorption in liquid alloys provide unique insight on interatomic interactions at the surface. The observed composition modulation could be accounted for quantitatively by the Defay-Prigogine and Strohl-King multilayer extensions of the single-layer Gibbs model, revealing a near-surface domination of the attractive Bi-Sn interaction over the entropy.
\end{abstract}

DOI: 10.1103/PhysRevLett.95.106103

PACS numbers: 68.03. $-\mathrm{g}, 61.10 .-\mathrm{i}, 61.20 .-\mathrm{p}$

The widely-accepted Gibbs adsorption rule [1] predicts the surface segregation of the lower surface energy component of a binary mixture. Liquid metals are ideal systems for studying Gibbs adsorption due to the nearly spherical shape of interacting particles, relative simplicity of the short-range interactions, and the availability of bulk thermodynamic data for many binary alloys. While certain aspects of Gibbs theory can be tested through macroscopic measurements of surface tension or adsorption isotherms, very few direct measurements of the atomic-scale composition profiles of the liquid-vapor interface were reported [2-4]. In addition to fundamental questions related to surface thermodynamics of binary liquids, Bi-Sn-based alloys have been widely studied as substitutes for $\mathrm{Pb}$-based low-melting solders [5]. Thus, understanding their wetting, spreading, alloying, reactivity, and other surface-related properties is of great practical importance. Moreover, interfacial phenomena dominate the properties of the increasingly important class of nanoscale materials, as demonstrated recently in studies of the liquid-solid phase stability of nanometer-sized Bi-Sn particles [6].

Synchrotron-based x-ray reflectivity (XR) can measure the surface-normal density profile of a liquid with Ångström-scale resolution. Over the last decade XR revealed the long-predicted surface-induced atomic layering at the liquid-vapor interface for a number of elemental liquid metals [7-11]. Resonant XR near an absorption edge resolved the density profile of each component in GaIn [2], $\mathrm{HgAu}$ [3] and BiIn [4] liquid binary alloys. The enhancement of the concentration of the low-surfacetension component was invariably found to be confined to the topmost surface monolayer, with subsequent layers having the composition of the bulk, in accord with the simplest, and widely used, interpretation of the Gibbs rule. By contrast, we find here an atomic-scale phase separation extending over at least three atomic layers.
This is unexpected, considering the near-perfect-solution nature of the $\mathrm{Bi}_{43} \mathrm{Sn}_{57}$ alloy in the bulk [12,13].

A liquid $\mathrm{Bi}_{43} \mathrm{Sn}_{57}$ sample (99.99\% purity, Alfa Aesar) was prepared under UHV conditions $\left(\mathrm{P}<10^{-9}\right.$ Torr $)$. Atomically clean liquid surfaces were obtained by mechanical scraping and $\mathrm{Ar}^{+}$ion sputtering, as described previously $[10,14,15]$. Measurements were done using the liquid surface diffractometer at the ChemMatCARS beamline, Advanced Photon Source, Argonne National Laboratory, at a sample temperature of $T=142{ }^{\circ} \mathrm{C}, 4^{\circ} \mathrm{C}$ above the $\mathrm{Bi}_{43} \mathrm{Sn}_{57}$ alloy's eutectic temperature, $T_{e}=$ $138^{\circ} \mathrm{C}$.

The reflected intensity fraction, $R\left(q_{z}\right)$, of an x-ray beam impinging on a structured liquid surface at a grazing angle $\alpha$, is given by the Born approximation as

$$
R\left(q_{z}\right)=R_{F}\left(q_{z}\right)\left|\Phi\left(q_{z}\right)\right|^{2} \mathrm{CW}\left(q_{z}\right)
$$

where $q_{z}=(4 \pi / \lambda) \sin \alpha, \quad \lambda$ is the x-ray wavelength, $R_{F}\left(q_{z}\right)$ is the Fresnel $\mathrm{XR}$ of an ideally abrupt and flat surface, $\mathrm{CW}\left(q_{z}\right)$ is due to thermal surface capillary waves $[9,10]$, and the surface's structure factor is $[16]$

$$
\Phi\left(q_{z}\right)=\frac{1}{\rho_{\infty}} \int d z \frac{d\langle\rho(z)\rangle}{d z} \exp \left(\imath q_{z} z\right)
$$

Here $z$ is the surface-normal axis, $\rho_{\infty}$ and $\rho(z)$ are the bulk and surface electron densities, respectively, and $\langle\ldots\rangle$ denotes surface-parallel averaging. As $R_{F}$ is a universal function depending only on the known critical angle for total external reflection, and $\mathrm{CW}\left(q_{z}\right)$ is known accurately from capillary wave theory, the intrinsic density profile $\langle\rho(z)\rangle$ is obtained by computer fitting the measured $R\left(q_{z}\right)$ by a physically motivated model described below [10].

The (forward) atomic scattering factor of a $Z$-electron atom varies with energy as [16]: $Z^{\prime}=Z+f^{\prime}(E)-i f^{\prime \prime}(E)$, where $f^{\prime}(E)$ and $f^{\prime \prime}(E)=\mu(E) \lambda /(4 \pi)$, are the real and 
imaginary parts of the dispersion correction, and $\mu(E)$ is the photoelectric absorption coefficient.

The effect of $f^{\prime \prime}$ on the analysis can be neglected and the changes in $f^{\prime}$ are significant only near an absorption edge. Figure 1(a) shows $f^{\prime \prime}(E)$ near the Bi L3 edge as obtained from an absorption measurement in a Bi foil. Figure 1(b) is the corresponding $f^{\prime}(E)$, calculated from $f^{\prime \prime}(E)$ using the Kramers-Kronig relation [17]. Both agree well with theory $[18,19]$. The composition dependence of $\langle\rho(z)\rangle$ was obtained by fitting the measured XR by the distorted crystal (DC) model for a layered liquid surface $[7,8]$ :

$$
\frac{\langle\rho(z)\rangle}{\rho_{\infty}}=\sum_{n=1}^{\infty} \frac{e^{-(z-n d)^{2} / \sigma_{n}^{2}}}{\sqrt{2 \pi} \sigma_{n} / d}\left(1+\delta_{n} \frac{Z_{\mathrm{Bi}}^{\prime}-Z_{\mathrm{Sn}}^{\prime}}{Z_{\infty}^{\prime}}\right) \frac{c_{n}}{c}
$$

The progressive increase in the Gaussian width parameter $\sigma_{n}^{2}=\sigma_{0}^{2}+(n-1) \bar{\sigma}^{2}$ with increasing layer number $n$ describes the layering amplitude's decay below the surface [8]. The layer spacing $d$ is kept constant in this model due to similarity in size between Bi and Sn atoms. The bulk's average effective electron number per atom is $Z_{\infty}^{\prime}=$ $x Z_{\mathrm{Bi}}^{\prime}+(1-x) Z_{\mathrm{Sn}}^{\prime}$, and $\delta_{n}=x_{n}^{\prime}-x$ is the difference in the Bi fraction between the $n$th layer, $x_{n}^{\prime}$, and the bulk, $x$. The corresponding atomic densities, $c_{n}$ and $c$, are determined from the atomic volumes $v_{\mathrm{Bi}}$ and $v_{\mathrm{Sn}}: c_{n} x_{n}^{\prime} v_{\mathrm{Bi}}+$ $c_{n}\left(1-x_{n}^{\prime}\right) v_{\mathrm{Sn}}=1$. The contrast, $\left(Z_{\mathrm{Bi}}^{\prime}-Z_{\mathrm{Sn}}^{\prime}\right) / Z_{\infty}^{\prime}$, varies strongly near the edge due to the variation of $Z_{\mathrm{Bi}}^{\prime}$ : from 0.43 at $E=12.00 \mathrm{keV}$ to 0.27 at $E=13.418 \mathrm{keV}$ (right axis in Fig. 1). This is the basis for the resonant XR method which allows us to separate out the density profiles of the two species $[4,15]$.

Figure 2 shows Fresnel-normalized XRs $R\left(q_{z}\right) / R_{F}\left(q_{z}\right)$ measured near the Bi L3 edge at the four energies marked

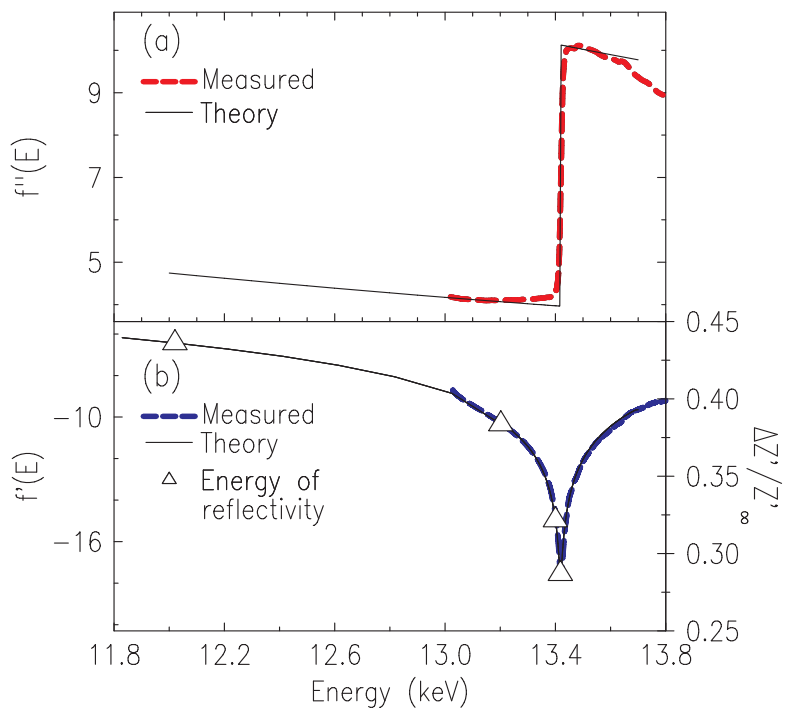

FIG. 1 (color online). Dispersion corrections (a) $f^{\prime}(E)$ and (b) $f^{\prime \prime}(E)$ of $\mathrm{Bi}$ near the $\mathrm{L} 3$ absorption edge at $E_{\mathrm{L} 3}=$ $13.418 \mathrm{keV}$. The right scale of (b) is the electron density contrast $\Delta Z^{\prime} / Z_{\infty}^{\prime}=\left(Z_{\mathrm{Bi}}^{\prime}-Z_{\mathrm{Sn}}^{\prime}\right) / Z_{\infty}^{\prime}$. by triangles in Fig. 1(b). The dashed line is calculated from the DC model for a layered interface assuming a uniform composition $\left(\delta_{n}=0\right)$. The strong enhancement of the measured $R\left(q_{z}\right) / R_{F}\left(q_{z}\right)$ over this line, evidenced by the peak at $q_{z} \simeq 1.0 \AA^{-1}$, and the strong energy dependence of the low- $q_{z}$ reflectivity, clearly indicate a significant surface segregation of $\mathrm{Bi}$, and its variation with $z$.

Three fits of the data by the DC model, Eq. (3), were carried out, assuming that only one $\left(\delta_{1} \neq 0, \delta_{n \geq 2}=0\right)$, two $\left(\delta_{1,2} \neq 0, \delta_{n \geq 3}=0\right)$, or three $\left(\delta_{1,2,3} \neq 0, \delta_{n \geq 4}=0\right)$ surface layers deviate from the bulk composition. All fits employed $d=2.90 \AA, \sigma_{0}=0.30 \AA$, and $\bar{\sigma}=0.57 \AA$, derived from the energy-independent position, shape and intensity of the layering peak at $q_{z}=2.0 \AA^{-1}$. The measured $R\left(q_{z}\right) / R_{F}\left(q_{z}\right)$ of all four energies were fitted simultaneously, using the experimentally determined $f^{\prime}(E)$.

Figure 2 exhibits an excellent agreement of the threelayer model (solid lines) with the measurements, but a very poor agreement for the one- and two-layer models (inset). Table I lists the best-fit values of $x_{n}^{\prime}$ and $\delta_{n}^{\mathrm{Fit}}=x_{n}^{\prime}-x$ and the corresponding 95\% nonlinear confidence intervals $Y\left(x_{n}^{\prime}\right)$ and $Y\left(\delta_{n}^{\mathrm{Fit}}\right)$ determined from a six-parameter support plane analysis [20]. The most striking result is the nonmonotonic deviation $x_{n}^{\prime}$ of Bi from the $43 \%$ bulk value, showing an enhanced composition of $96 \%$ and $53 \%$ in the first and third layers, and depletion down to $25 \%$ in the second layer (see Fig. 3). Beyond the third layer entropy effects dominate the Gibbs adsorption and the layer and bulk concentrations cannot be distinguished. While demixing has not been previously reported in liquid alloys, similar decaying oscillatory composition profiles were dis-

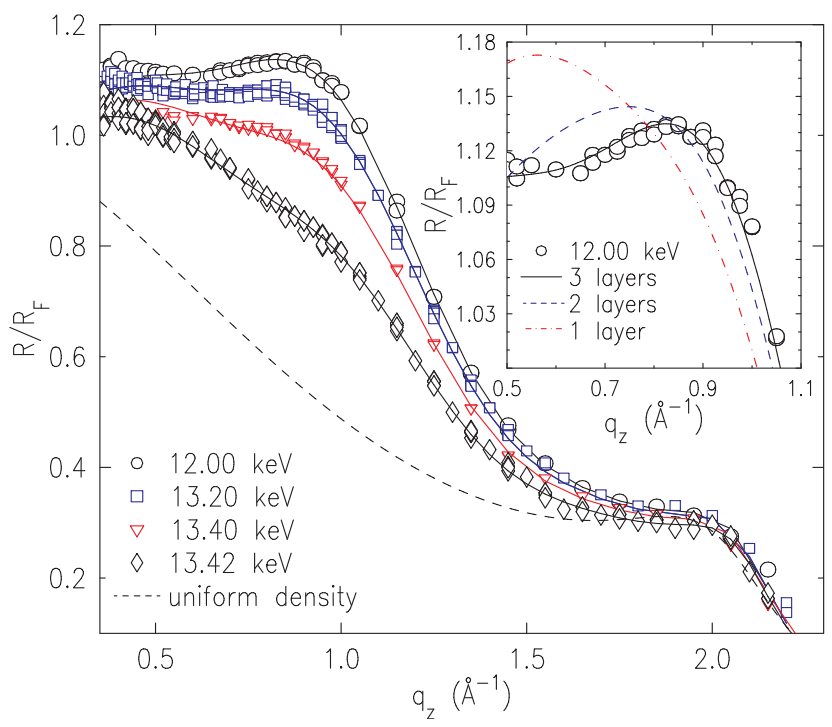

FIG. 2 (color online). XR measured at the indicated energies, with fits by the three-layer model (lines). The dashed line is the $\mathrm{XR}$ of a uniform-composition surface. Inset: The $E=$ $12.00 \mathrm{keV}$ measured $R / R_{F}$ with fits by the three models discussed in the text (lines). Error bars are smaller than the symbols' size. 
TABLE I. Density model parameters $x_{n}^{\prime}$ and $\delta_{n}^{\text {Fit }}=x_{n}^{\prime}-x$, and confidence intervals $Y\left(x_{n}^{\prime}\right)$ and $Y\left(\delta_{n}^{F i t}\right)$ obtained from the three-layer model fits compared to theoretical $\delta_{n}^{\mathrm{DP}}$ and $\delta_{n}^{\mathrm{SK}}$ derived from the Defay-Prigogine and Strohl-King models.

\begin{tabular}{ccccccc}
\hline \hline$n$ & $x_{n}^{\prime}(\mathrm{Bi})$ & $Y\left(x_{n}^{\prime}\right)$ & $\delta_{n}^{\mathrm{Fit}}$ & $Y\left(\delta_{n}^{\mathrm{Fit}}\right)$ & $\delta_{n}^{\mathrm{DP}}$ & $\delta_{n}^{\mathrm{SK}}$ \\
\hline 1 & 0.96 & {$[0.94,0.99]$} & 0.53 & {$[0.51,0.56]$} & 0.47 & 0.51 \\
2 & 0.25 & {$[0.18,0.27]$} & -0.18 & {$[-0.25,-0.16]$} & -0.23 & -0.25 \\
3 & 0.53 & {$[0.50,0.56]$} & 0.10 & {$[0.07,0.13]$} & 0.12 & 0.05 \\
4 & 0.43 & $\cdots$ & 0 & $\cdots$ & -0.06 & -0.01 \\
\hline \hline
\end{tabular}

covered in several crystalline alloys such as $\mathrm{Cu}_{3} \mathrm{Au}$ [21]. However, the properties, formation mechanism, and strong temperature dependence of the composition modulations in $\mathrm{Cu}_{3} \mathrm{Au}$ alloys were found to be intimately related to, and largely dominated by, the long-range fcc order in the bulk crystal, and the severe packing strains resulting from the big mismatch in the atomic diameter of the two components. As none of these exist in our liquid alloy, surfaceinduced segregation and the Gibbs rule can be studied in a pure short-range interaction context, free from the complicating influence, or even dominance, of other effects. We now compare our experimental observations with theory.

Guggenheim's [22] application of Gibbs theory [1] to regular solutions assumes the surface segregation to be restricted to a single surface monolayer. Assuming $p$ nearest neighbors for each bulk atom in a layered lattice model, $l p$ are within, and $m p$ are in the adjacent, layers. For a close-packed lattice, for example, $p=12, l=0.5$, and $m=0.25$. The surface tension of the regular solution, $\gamma_{A B}$, follows from those of the pure components, $\gamma_{A}$ and $\gamma_{B}$, as [22]

$$
\begin{aligned}
\gamma_{A B} & =\gamma_{B}+\frac{k T}{a_{B}} \ln \left(\frac{1-x^{\prime}}{1-x}\right)+\frac{\omega}{a_{B}}\left[l x^{\prime 2}-(l+m) x^{2}\right] \\
& =\gamma_{A}+\frac{k T}{a_{A}} \ln \left(\frac{x^{\prime}}{x}\right)+\frac{\omega}{a_{A}}\left[l\left(1-x^{\prime}\right)^{2}-(l+m)(1-x)^{2}\right] .
\end{aligned}
$$

Here, $x$ and $(1-x)$ are the bulk concentrations of atoms $A$ (Bi) and $B(\mathrm{Sn})$, while $x^{\prime} \neq x$ and $\left(1-x^{\prime}\right)$ are the corresponding surface concentrations, $a_{A}$ and $a_{B}$ are the two atomic areas, and $\omega=2 \omega_{A B}-\omega_{A A}-\omega_{B B}$ is the interaction parameter, defined by the $A-B, A-A$, and $B-B$ atomic interaction energies. Extrapolated down to $T=142{ }^{\circ} \mathrm{C}$, $\gamma_{\mathrm{Bi}}=398 \mathrm{mN} / \mathrm{m}$, and $\gamma_{\mathrm{Sn}}=567 \mathrm{mN} / \mathrm{m}$, while $a_{\mathrm{Bi}}$ and $a_{\mathrm{Sn}}$ are calculated from the atomic radii $r_{\mathrm{Bi}}=1.70 \AA$ and $r_{\mathrm{Sn}}=1.62 \AA$ assuming hexagonal close packing [23]. Treating $\mathrm{Bi}_{43} \mathrm{Sn}_{57}$ as a perfect solution $(\omega / k T=0)$, the Gibbs theorem, Eq. (4), yields $\gamma_{A B}=444 \mathrm{mN} / \mathrm{m}$ and $x^{\prime}=$ 0.904 , below the experimental value $x_{1}^{\prime}(\mathrm{Bi})$ in Table I. However, assuming a regular solution behavior with $\omega / k T=1$ yields $\gamma_{A B}=432 \mathrm{mN} / \mathrm{m}$, and $x^{\prime}=0.941$, which agrees very well with the experimentally derived $x_{1}^{\prime}(\mathrm{Bi})$ in Table I. Both $\gamma_{A B}$ agree well with experiment and theory [24]. Note that $\gamma_{A B}$ and $x^{\prime}$ are only weakly depen-

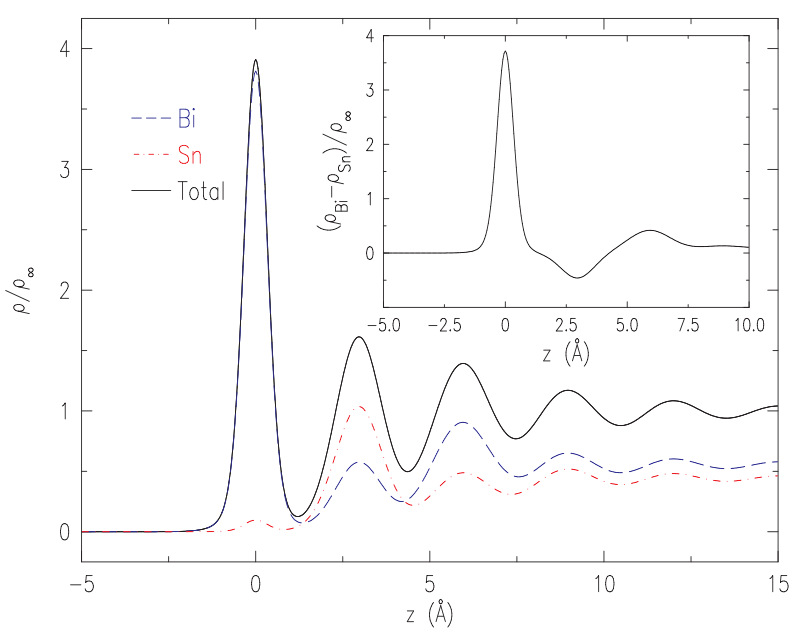

FIG. 3 (color online). Electron density profiles as derived from the fits to the reflectivities shown in Fig. 2. Inset: the bulknormalized differences in electron density of Bi and $\mathrm{Sn},\left(\rho_{\mathrm{Bi}}-\right.$ $\left.\rho_{\text {Sn }}\right) / \rho_{\infty}$.

dent on $\omega / k T$ due primarily to the logarithmic functional behavior and large surface tension difference of the two components, $a_{\mathrm{Sn}} \gamma_{\mathrm{Sn}}-a_{\mathrm{Bi}} \gamma_{\mathrm{Bi}} \approx 2 k T$. This introduces a large uncertainty of $\omega / k T$ calculated from measurements of surface tension or surface monolayer composition. Resonant XR measurements of subsurface layer composition therefore present a unique opportunity to probe the nature of atomic interactions at the surface.

In spite of the good agreement above, confining the surface excess to a single monolayer is correct for perfect solutions only, but not for our case of a regular solution, as Defay and Prigogine [25] point out. They provide a correction for regular solutions, where the surface excess extends over two layers, the $\gamma_{A B}$ values above do not change significantly, and the layers' $\delta_{n}$ are related by

$$
\ln \frac{1+\delta_{2} / x}{1-\delta_{2} /(1-x)}-\frac{2 \omega}{k T} \delta_{2}-\frac{2 \omega m}{k T}\left(\delta_{1}-2 \delta_{2}\right)=0 .
$$

Expanding Eq. (5) to first order in $\delta_{2}$ :

$$
\delta_{2}=\frac{2 \omega m x(1-x) \delta_{1}}{k T-2 \omega l x(1-x)} .
$$

For nearly perfect solutions $(\omega / k T \ll 1)$ Eq. (5) yields a negligible $\delta_{2}: 0<\delta_{2} \ll \delta_{1}$. For $\omega / k T \geqslant 1$, however, $\delta_{2}$ and $\delta_{1}$ are of opposite signs and $\left|\delta_{2}\right|$ may become comparable to $\left|\delta_{1}\right|$. This prediction is qualitatively consistent with the demixing observed here. For example, when $\omega / k T \gg 1$, Eq. (6) can be simplified further: $\delta_{2}=$ $-(m / l) \delta_{1}$. For $\mathrm{Bi}_{43} \mathrm{Sn}_{57}, m / l \approx 0.5$ and the Gibbspredicted $x_{1}^{\prime}=0.90$ (or $\delta_{1}=0.47$ ) yields $\delta_{2}=-0.23$, $\delta_{3}=0.12$, and $\delta_{4}=-0.06$ [26]. These values, shown as $\delta_{n}^{\mathrm{DP}}$ in Table I, agree well with $\delta_{n}^{\mathrm{Fit}}$ obtained from the threelayer model fits. The smallest value of the interaction parameter $\omega / k T$ for which satisfactory agreement with 
the Defay-Prigogine model could be obtained (by treating $m$ as an adjustable parameter) is $\omega / k T=2.3$. Strohl and King [27] suggest a multilayer, multicomponent model, where no expansion is used, and $x_{n}^{\prime}$ are obtained iteratively, until convergence to a self-consistent composition profile is reached. A good agreement of this theory with our Bi-Sn measurements is obtained when $\omega / k T=1 \cdot 0-1.7$. Typical $\delta_{n}^{\mathrm{SK}}$ values are listed in Table I. As observed, the StrohlKing model provides composition profiles very similar to those of the Defay-Prigogine model, albeit with slightly different $\delta_{n}$ values, thus supporting our overall conclusions.

Theoretically, $\omega$ and the enthalpy of mixing, $\Delta H_{m}$, are related by $\omega=\Delta H_{m} /[x(1-x)]$. In practice, however, bulk thermodynamic quantities were often found to yield inaccurate values for surface quantities. For example, organic [28] and metallic [29] mixtures exhibit significant disagreements between $\omega$ values derived empirically from surface tension measurements and from bulk calorimetry. For Bi-Sn, reported values of $\Delta H_{m}$ range from endothermic values of 80 to $140 \mathrm{~J} / \mathrm{mol}$ [12] to an exothermic value of $-180 \mathrm{~J} / \mathrm{mol}$ [13]. These values lead to $|\omega / k T|<0.2$, i.e., an almost perfect solution, and an insignificant $\left|\delta_{2}\right|<$ 0.01 . On the other hand, the value of $\omega / k T \approx 10$ that we previously found necessary to account for the observed $35 \% \mathrm{Bi}$ concentration enhancement at the surface monolayer at the BiIn eutectic is of the same order of magnitude as the value we find necessary to account for the present observation of surface segregation in $\mathrm{Bi}-\mathrm{Sn}, \omega / k T \approx$ 1.0-2.3. Unfortunately we do not have an explanation for the origin of the discrepancy in the values of $\omega / k T$ and this suggests an urgent need for both further theoretical studies of surface demixing as well as experimental investigations of similar effects in other binary alloys. In particular, the Bi-Sn system appears to be the only liquid alloy for which clear evidence for multilayer surface demixing has been found. The case for new studies is strongly reinforced by the existence of a growing class of surface-induced ordering phenomena that have been observed in metallic liquids. In addition to the surface demixing reported here, these include layering [7-11], relaxation [11], segregation [2$4,30]$, wetting transitions [14,31], and surface freezing [32]. Finally, there is a basic unresolved question of whether the surfaces of liquid metals are fundamentally different from those of nonmetallic liquids [33].

This work has been supported by the U.S. DOE Grants No. DE-FG02-88-ER45379 and No. DE-AC0298CH10886 and the U.S.-Israel Binational Science Foundation, Jerusalem. We gratefully acknowledge useful discussions with E. Sloutskin at Bar-Ilan as well as assistance from T. Graber, D. Schultz, and J. Gebhardt at ChemMatCARS Sector 15, principally supported by the NSF/DOE Grant No. CHE0087817. The Advanced Photon Source is supported by the U.S. DOE Contract No. W-31109-Eng-38.
*Electronic address: oleg@xray.harvard.edu Present address: Center for Nanoscale Materials, Argonne National Laboratory, Argonne, IL, 60439.

[1] J. W. Gibbs et al., The Collected Works of J. Willard Gibbs (Longmans, New York, 1928).

[2] M. J. Regan et al., Phys. Rev. B 55, 15874 (1997).

[3] E. DiMasi et al., Mater. Res. Soc. Symp. Proc. 590, 183 (2000).

[4] E. DiMasi et al., Phys. Rev. Lett. 86, 1538 (2001).

[5] K. N. Tu et al., J. Appl. Phys. 93, 1335 (2003); J. Glazer, Int. Mater. Rev. 40, 65 (1995).

[6] J. G. Lee and H. Mori, Phys. Rev. B 70, 144105 (2004).

[7] O. M. Magnussen et al., Phys. Rev. Lett. 74, 4444 (1995).

[8] M. J. Regan et al., Phys. Rev. Lett. 75, 2498 (1995).

[9] H. Tostmann et al., Phys. Rev. B 59, 783 (1999).

[10] O. G. Shpyrko et al., Phys. Rev. B 67, 115405 (2003).

[11] O. G. Shpyrko et al., Phys. Rev. B 70, 224206 (2004).

[12] N. A. Asryan and A. Mikula, Inorg. Mater. 40, 386 (2004); R. Hultgren et al., Selected Values of the Thermodynamic Properties of Binary Alloys (Metals Park, OH, 1973); R. L. Sharkey and M. J. Pool, Metall. Trans. 3, 1773 (1972); H. Seltz et al., J. Am. Chem. Soc. 64, 1392 (1942).

[13] S. A. Cho and J. L. Ochoa, Metall. Mater. Trans. B 28, 1081 (1997).

[14] P. Huber et al., Phys. Rev. Lett. 89, 035502 (2002).

[15] O. G. Shpyrko, Ph.D. thesis, Harvard University, 2003.

[16] J. Als-Nielsen and D. McMorrow, Elements of Modern $X$-Ray Physics (Wiley, New York, 2001).

[17] G. Evans and R.F. Pettifer, J. Appl. Crystallogr. 34, 82 (2001); J. J. Hoyt et al., J. Appl. Crystallogr. 17, 344 (1984).

[18] E. A. Merritt, Anomalous Scattering Coefficients, http:// www.bmsc.washington.edu/scatter/ (1996).

[19] M. Newville, computer code IFEFFIT, 1997.

[20] P. R. Bevington and D. K. Robinson, Data Reduction and Error Analysis for the Physical Sciences (McGraw-Hill, New York, 1992) p. 212.

[21] H. Reichert et al., Phys. Rev. Lett. 78, 3475 (1997); 74, 2006 (1995); 90, 185504 (2003).

[22] E. A. Guggenheim, Trans. Faraday Soc. 41, 150 (1945).

[23] CRC Handbook of Chemistry and Physics, edited by D. R. Lide (CRC, Boca Raton, 1996).

[24] S.Z. Yoon et al., Scr. Mater. 40, 297 (1999).

[25] R. Defay and I. Prigogine, Trans. Faraday Soc. 46, 199 (1950).

[26] Equation (6) yields $\delta_{3}$ from $\delta_{2}$ and $\delta_{4}$ from $\delta_{3}$.

[27] J. K. Strohl and T. S. King, J. Catal. 118, 53 (1989).

[28] G. L. Gaines, Trans. Faraday Soc. 65, 2320 (1969).

[29] T. P. Hoar and D. A. Melford, Trans. Faraday Soc. 53, 315 (1957).

[30] E. B. Flom et al., Science 260, 332 (1993).

[31] H. Shim et al., Surf. Sci. 476, L273 (2001); A. H. Ayyad and W. Freyland, ibid. 506, 1 (2002).

[32] A. Issanin et al., J. Chem. Phys. 121, 12005 (2004); B. Yang et al., Phys. Rev. B 62, 13111 (2000); O. G. Shpyrko et al. (to be published).

[33] E. Chacón and P. Tarazona, Phys. Rev. Lett. 91, 166103 (2003); D. X. Li and S. A. Rice, J. Phys. Chem. B 108, 19640 (2004); O. G. Shpyrko et al., Phys. Rev. B 69, 245423 (2004). 